\title{
The $\alpha-\gamma-\varepsilon$ triple point of iron investigated by high pressure-high temperature neutron scattering
}

\author{
S. Klotz, ${ }^{1, a)}$ Y. Le Godec, ${ }^{1}$ Th. Strässle, ${ }^{2}$ and U. Stuhr ${ }^{2}$ \\ ${ }^{1}$ IMPMC, Université P\&M Curie, 4 Place Jussieu, 75252 Paris, France \\ ${ }^{2}$ Laboratory for Neutron Scattering, ETH Zurich \& Paul Scherrer Institut, 5232 Villigen PSI, Switzerland
}

(Received 16 July 2008; accepted 30 July 2008; published online 3 September 2008)

\begin{abstract}
We present high pressure-high temperature diffraction data in the 0-10 GPa and 300-1000 K range of all three main iron phases. The refinements of the diffraction patterns give molar volumes to high precision and show that the $\alpha-\gamma-\varepsilon$ triple point is located at $8.2 \pm 0.1 \mathrm{GPa}$ and $678 \mathrm{~K}$. These values are significantly lower than currently admitted $(10.4 \mathrm{GPa} / 740 \mathrm{~K})$, but are consistent with reported in situ X-ray diffraction data obtained in multianvil presses. These measurements have been made possible by the use of a high P/T cell which uses metallic toroidal gaskets and which suffers from less absorption, gives clean diffraction patterns, and shows better pressure performance than other setups. () 2008 American Institute of Physics. [DOI: 10.1063/1.2976128]
\end{abstract}

Iron has three solid phases: ${ }^{1}$ the ambient-condition bcc phase ( $\alpha$-iron), the high temperature fcc phase ( $\gamma$-iron), and the high pressure hcp phase ( $\varepsilon$-iron). All three phases have been investigated in detail from the early 1950s on when experimental techniques became available which allowed measurements at pressures and temperatures to $15 \mathrm{GPa}$ and $1000 \mathrm{~K}$. From the compiled data obtained by resistivity measurements in a belt apparatus it was concluded that the $\alpha-\gamma-\varepsilon$ triple point is located at $10.4 \mathrm{GPa}$ and $740 \mathrm{~K} .^{1,2}$ Although at least one later in situ $\mathrm{x}$-ray diffraction measurement reported considerably different results, ${ }^{3}$ these values became commonly accepted reference data. The issue is of considerable importance since iron is the most abundant metal on earth and the sixth most abundant element in the universe. ${ }^{1}$ As such, its thermodynamic properties are important for various fields ranging from engineering to fundamental physics and earth sciences.

Here we report in situ neutron diffraction data on the $\alpha-\gamma-\varepsilon$ triple point of iron. Compared to previous experiments ${ }^{4,5}$ our approach has several novelties. The experiments provide clean diffraction data which can be readily refined to give molar volumes with unprecedented precision. The temperature gradients in our setup are shown to be negligible, and the temperature is measured by a neutronresonance technique which does not rely on thermocouples.

The measurements were made possible by the development of a high P/T setup for the Paris-Edinburgh press using opposed anvils as shown in Fig. 1. High temperatures are generated by resistive heating using a graphite furnace and an electric current of typically $150 \mathrm{~A}$ fed through the two anvils. The essential difference compared to previously described methods ${ }^{4,5}$ is the use of metallic toroidal gaskets. To prevent a short circuit the toroidal gasket is split in two halves with an $\mathrm{MgO}$ layer between. Efficient background reduction is achieved by careful collimation of the incident beam as well as ${ }^{10} \mathrm{~B}$ coating of the stainless steel contacts shown in Fig. 1. Extensive finite element calculations were carried out to optimize the dimensions and the choice of materials. An important result of these calculations is that the temperature gradients across a sample with a thermal con-

${ }^{\text {a)} E l e c t r o n i c ~ m a i l: ~ S t e f a n . K l o t z @ i m p m c . j u s s i e u . f r . ~}$ ductivity of pure iron $(80 \mathrm{~W} / \mathrm{mK})$ cannot be larger than $\pm 3 \mathrm{~K}$ at $1000 \mathrm{~K}$. All measurements during the development phase were carried out at the POLDI diffractometer of the SINQ spallation source located at the Paul Scherrer Institute, Villigen, Switzerland, using a VX-type Paris-Edinburgh load-frame. ${ }^{6}$ POLDI is a strain diffractometer which allows precise measurements of lattice parameters at a constant diffraction angle of $90^{\circ}$ sampling a volume of $1 \mathrm{~mm}^{3}$, thus eliminating all signal from the sample environment. The final measurements reported here were carried out at the PEARL station of the U.K. ISIS Facility at Chilton, Didcot. A description of the general setup for high-P/T measurements can be found in Refs. 5 and 7. A key element is the possibility of determining the temperature in situ by neutron-resonance absorption measurement. ${ }^{7}$ As demonstrated previously, ${ }^{5,7}$ the width of the absorption lines of a $50 \mu \mathrm{m}$ Ta foil embedded in the sample (Fig. 1) is directly related to the temperature via the Doppler effect. From numerous measurements during

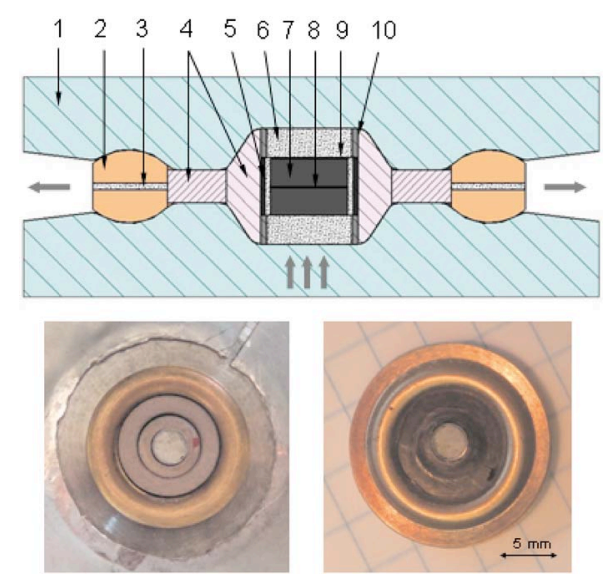

FIG. 1. (Color online) Gasket-anvil assembly for high P/T neutron diffraction used in the experiments. (1) anvil, (2) toroidal gasket (CuBe), (3) $\mathrm{MgO}$ layer, (4) baked phyrophillite, (5) graphite furnace, (6) MgO disk, (7) sample (iron), (8) Ta foil, (9) Hf foil, (10) electrical contacts (stainless steel). The sample (Fe, $99.99 \%$ purity from Alfa Aesar) consists of two cylindrical rods of $3.5 \mathrm{~mm}$ diameter with the $50 \mu \mathrm{m}$ Ta foil between them. The directions of incident and diffracted beams are indicated by thick gray arrows. The photographs below show the assembly from above, before (left) and after (right) the experiment. 


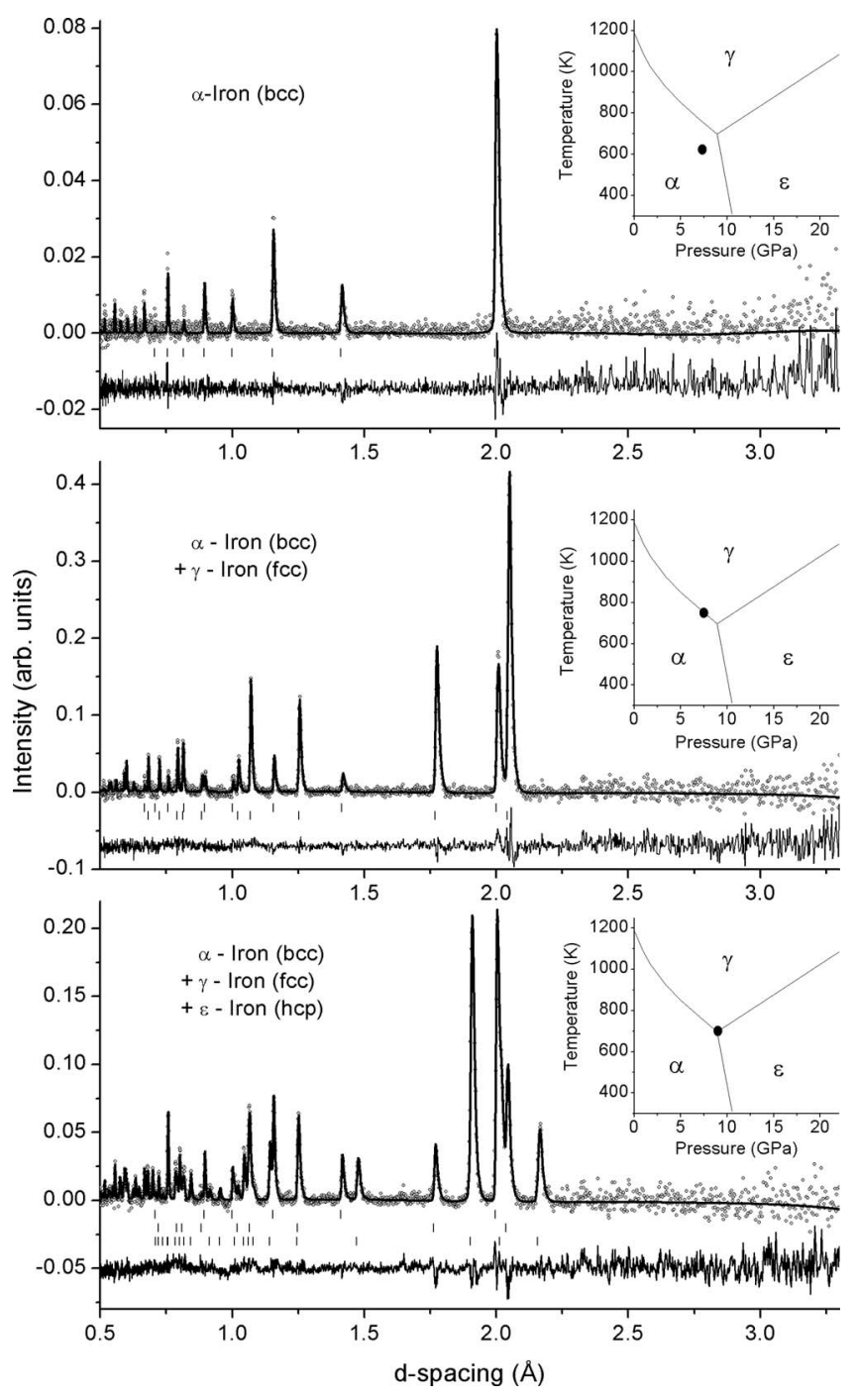

FIG. 2. Neutron diffraction patterns of iron at three different P/T conditions as indicated in the inset next to each spectrum. The line is a fit to the data (dots), difference plots and tick marks are given below (lower panel: upper, middle and lower tick marks correspond to $\alpha-\gamma$ - and $\varepsilon$-phases, respectively). The accumulation times are $\sim 11 \mathrm{~min}(35 \mu \mathrm{Ah}), \sim 55 \mathrm{~min}$ $(180 \mu \mathrm{Ah})$ and $\sim 110 \mathrm{~min}(360 \mu \mathrm{Ah})$ for the upper, middle and lower pattern, respectively.

the past five years, the accuracy of this temperature measurement is established to be $\pm 20 \mathrm{~K}$ at $1000 \mathrm{~K}$. We confirmed this accuracy (and precision) with a second foil made of $\mathrm{Hf}$ (thickness also $50 \mu \mathrm{m}$ ) which has resonance lines at different energies and which gave in almost all of the 40 measured $\mathrm{P} / \mathrm{T}$ points identical temperatures within these limits.

Figure 2 shows diffraction patterns obtained at various $\mathrm{P} / \mathrm{T}$ conditions including fits by Rietveld refinements. Remarkable is the data quality and absence of any contaminant signal from the sample environment. We estimate that beyond $5 \mathrm{GPa}$, the use of the cell shown in Fig. 1 gives intensities at least two times higher than previously possible using the setup based on conoidal anvils. ${ }^{5,7}$ This is due to the observation that, even at high load, the gap between the two toroidal anvils remains appreciable $(0.5 \mathrm{~mm})$, whereas it rapidly decreases in conoidal opposed anvils. The pressure performance of the setup is considerable; as far as we are aware of, none of the high P/T cells used so far for neutron scattering has reached such elevated pressures. The temperature was cycled several times between 300 and $1300 \mathrm{~K}$ at loads
TABLE I. Molar volumes of $\alpha-, \gamma-$ and $\varepsilon$-iron at various $\mathrm{P} / \mathrm{T}$ conditions (values in brackets denote corresponding volume phase fractions).

\begin{tabular}{lccc}
\hline \hline & $\begin{array}{c}V_{\alpha} \\
\left(\mathrm{cm}^{3} / \mathrm{mol}\right)\end{array}$ & $\begin{array}{c}V_{\gamma} \\
\left(\mathrm{cm}^{3} / \mathrm{mol}\right)\end{array}$ & $\begin{array}{c}V_{\varepsilon} \\
\left(\mathrm{cm}^{3} / \mathrm{mol}\right)\end{array}$ \\
\hline $\begin{array}{l}\alpha-\gamma-\varepsilon \text { triple point } \\
0 \mathrm{GPa} / 1180 \mathrm{~K}^{\mathrm{b}}\end{array}$ & $\begin{array}{c}6.81(19 \%) \\
7.37\end{array}$ & $\begin{array}{c}6.64(30 \%) \\
7.30\end{array}$ & $6.54(51 \%)$ \\
$9.2 \mathrm{GPa} / 300 \mathrm{~K}^{\mathrm{c}}$ & $6.76(83 \%)$ & & $6.44(17 \%)$ \\
\hline \hline
\end{tabular}

${ }^{\mathrm{a}}$ This work: $T=678 \mathrm{~K}, P=8.2 \mathrm{GPa}$.

${ }^{\mathrm{b}}$ Reference 2 .

${ }^{\mathrm{c}}$ This work.

up to 130 tons with only a slight reduction in efficiency of temperature generation. After the experiment the gasketsample assembly was recovered as shown in the inset of Fig. 1 , the anvils were undamaged. A considerable advantage of such metallic toroidal gaskets is that under load they deform plastically and do not fracture and crumble (see Fig. 1 ) as it is observed in assemblies made entirely of non-metallic materials (Refs. 4 and 5). This insures a reproducible gasket/ sample geometry and hence more reliable absorption corrections.

Figure 2 (lower panel) gives a diffraction pattern with all three iron phases present, i.e., obtained exactly at the $\alpha-\gamma-\varepsilon$ triple point. From the refinement we obtain the most precise molar volumes so far reported: $V_{\alpha}=6.81 \mathrm{~cm}^{3} / \mathrm{mol}$ (11.31 $\AA^{3} /$ atom) $, V_{\gamma}=6.64 \mathrm{~cm}^{3} / \mathrm{mol}\left(11.03 \AA^{3} /\right.$ atom $), V_{\varepsilon}$ $=6.54 \mathrm{~cm}^{3} / \mathrm{mol}\left(10.86 \AA^{3} /\right.$ atom $)$; see Table I. Hence, at the triple point, the molar volume of $\alpha$-Fe is $0.17 \mathrm{~cm}^{3} / \mathrm{mol}$ larger than that of $\gamma-\mathrm{Fe}$, and $0.27 \mathrm{~cm}^{3} / \mathrm{mol}$ larger than that of $\varepsilon$-iron. These values agree within $10 \%$ with those derived by extrapolating data from in situ x-ray measurements by Besson et al. ${ }^{8}\left(0.20 \mathrm{~cm}^{3} / \mathrm{mol}\right.$ and $0.30 \mathrm{~cm}^{3} / \mathrm{mol}$, respectively). Table I compares the differences in molar volumes at the triple point with those measured at ambient pressure and high temperature ( $\alpha-\gamma$ transition) as well as at high pressure and ambient temperature $(\alpha-\varepsilon$ transition). For the latter we find from a data point containing a mixture of $\alpha$ - and $\varepsilon$-iron a volume difference of $0.32 \mathrm{~cm}^{3} /$ mole at $300 \mathrm{~K}$ and 9.2 GPa. On the other hand, the difference in molar volumes between $\alpha$ and $\gamma$ iron at 1 bar and $1183 \mathrm{~K}$ is known to be $0.07 \mathrm{~cm}^{3} / \mathrm{mol}^{2}$. Hence, whereas the difference in molar volumes slightly decrease along the $\alpha-\varepsilon$ line, it strongly increases with pressure along the $\alpha-\gamma$ transition, i.e., the difference in density between $\alpha$ and $\gamma$-iron at the triple point is $\sim 2.5$ larger than at ambient pressure and high temperature. This is most likely due to a magnetoelastic effect in $\alpha$-iron: bcc-iron is paramagnetic at 1 bar at the $\alpha-\gamma$ phase boundary, whereas it is ferromagnetic at the $\alpha-\gamma-\varepsilon$ triple point. The ferromagnetic order in $\alpha$-Fe tends to expand the lattice and hence increases the difference in $V_{\alpha}$ and $V_{\gamma}$ as the pressure is increased. ${ }^{9}$

Although we did not use any pressure marker, ${ }^{10}$ the pressure values in our measurements are severely constrained by the measured molar volumes and the temperature, since the equations of state (EOS) of $\varepsilon$-Fe and $\alpha$-Fe are known to high precision. We use the thermoelastic EOS recently determined by Dewaele et al. ${ }^{11}$ which at $300 \mathrm{~K}$ is in excellent agreement with the EOS for $\alpha$-Fe determined both by ultrasonic measurements to $1 \mathrm{GPa}$ (Ref. 12) as well as by in situ $\mathrm{x}$-ray diffraction to $30 \mathrm{GPa} .{ }^{13}$ Given at the triple point a measured temperature of $678 \mathrm{~K}$ and a measured molar 
volume of $V_{\varepsilon}=6.54 \mathrm{~cm}^{3} / \mathrm{mol}$ (Table I), the pressure is constrained to be $8.2 \pm 0.1 \mathrm{GPa}$. This value is rather insensitive to a potential error in temperature reading, i.e., an error in 50 $\mathrm{K}$ would change the calculated pressure by only $0.3 \mathrm{GPa}$. We hence conclude that the pressure value of $10.4 \mathrm{GPa}$ so far admitted at the triple point is overestimated by approximately $20 \%$ (2 GPa). Such value would be strongly incompatible with our measured molar volumes and the published EOS of both $\varepsilon$ - and $\alpha$-iron, ${ }^{11-13}$ and can be safely excluded. Also, our triple point temperature $(678 \mathrm{~K})$ is $62 \mathrm{~K}$ lower than admitted so far. The source of the discrepancy in pressure is most likely the following. The textbook values for $P$ at the triple point ${ }^{1,2}$ were derived from thermodynamic fits to resistivity data by Bundy, ${ }^{14}$ obtained in a belt apparatus. The values cited in Ref. 14 resulted from a calibration curve based on various "fixed points," i.e., transition pressures of several elements, and then corrected to be consistent with data on the $\alpha-\varepsilon$ transition by Bancroft et al. ${ }^{15}$ which in turn were believed to be reliable since the $\alpha-\varepsilon$ transition pressure agreed with those determined by earlier shock wave measurements. Since the "fixed-point" pressures were redefined since then, and Bundy's pressure values relied on a series of assumptions, it is clear that the currently accepted location of the triple point is highly uncertain. Interestingly, our value agrees very well with in situ energy dispersive x-ray diffraction measurements using a large-volume cubic press, data which have not been promoted very much. ${ }^{3}$ Here the triple point was "tentatively located at $8.3 \mathrm{GPa}$ and $440{ }^{\circ} \mathrm{C}$," in excellent agreement with our neutron measurements. We note that both the $\mathrm{x}$-ray work $^{3}$ as well as our data give a triple point temperature lower than Refs. 1 and 2 by respectively 27 and $62 \mathrm{~K}$.

In conclusion, we suggest that the currently admitted location of the $\alpha-\gamma-\varepsilon$ triple point of iron should be reconsidered. Our in situ neutron measurements give $\mathrm{P}_{\alpha-\gamma-\varepsilon}$ $=8.2 \mathrm{GPa}$ and $\mathrm{T}_{\alpha-\gamma-\varepsilon}=678 \mathrm{~K}$, values which are supported by the $\mathrm{x}$-ray data of Akimoto et al. ${ }^{3}$
This work was partially based on experiments performed at the Swiss spallation neutron source SINQ, Paul Scherrer Institute, Villigen, Switzerland. We greatly acknowledge access to beamtime at the U.K. neutron facility ISIS. The authors thank M. Tucker (ISIS) for help in the experiments, as well as J. Philippe for assistance in the preparation of the measurements. The finite element calculations were carried out by L. Aloa-Eddine and G. Lin under the supervision of J. Frelat and S.K.

${ }^{1}$ D. Young, Phase Diagrams of the Elements (University of California Press, Berkeley, CA, 1991).

${ }^{2}$ A. F. Guillermet and P. Gustafson, High Temp. - High Press. 16, 591 (1985).

${ }^{3}$ S. Akimoto, T. Suzuki, T. Yagi, and O. Shimomura, in High-Pressure Research in Mineral Physics, edited by M. H. Manghnani and Y. Syono (Terra Scientific, Tokyo, 1987), p. 149.

${ }^{4}$ Y. Zhao, R. B. Von Dreele, and J. G. Morgan, High Press. Res. 16, 161 (1999).

${ }^{5}$ Y. Le Godec, M. T. Dove, D. J. Francis, S. C. Kohn, W. G. Marshall, A. R. Pawley, G. D. Price, S. A. T. Redfern, N. Rhodes, N. L. Ross, P. F. Schofield, E. Schooneveld, G. Syfosse, M. G. Tucker, and M. D. Welch, Miner. Mag. 65, 749 (2001).

${ }^{6}$ S. Klotz, G. Hamel, and J. Frelat, High Press. Res. 24, 219 (2004).

${ }^{7}$ H. J. Stone, M. G. Tucker, F. M. Méducin, M. T. Dove, S. A. T. Redfern, Y. Le Godec, and W. G. Marshall, J. Appl. Phys. 98, 064905 (2005).

${ }^{8}$ J. M. Besson and M. Nicol, J. Geophys. Res. 95, 21717 (1990).

${ }^{9}$ E. G. Moroni and T. Jarlborg, Europhys. Lett. 33, 223 (1996).

${ }^{10} \mathrm{The}$ use of a pressure marker such as $\mathrm{NaCl}$ would not have helped since the accuracy of the equation of state is not better known than that of $\varepsilon-\mathrm{Fe}$ (Ref. 11), i.e., the sample itself. Also, it has to be assumed that the marker is rigorously at the same pressure as the sample, an assumption which is not obvious due to the "Lamé effect," see Y. Wang, D. J. Weidner, and Y. Meng, in Properties of Earth and Planetary Materials at High Pressure and Temperature, edited by M. H. Manghnani and T. Yagi (AGU, Washington, DC, 1998), p. 365.

${ }^{11}$ A. Dewaele, P. Loubeyre, F. Occelli, M. Mezouar, P. I. Dorogokupets, and M. Torrent, Phys. Rev. Lett. 97, 215504 (2006).

${ }^{12}$ M. W. Guinan and D. N. Beshers, J. Phys. Chem. Solids 29, 541 (1968).

${ }^{13}$ H.-K. Mao, W. A. Bassett, and T. Takahashi, J. Appl. Phys. 38, 272 (1967).

${ }^{14}$ F. P. Bundy, J. Appl. Phys. 36, 616 (1965).

${ }^{15}$ D. Bancroft, E. L. Peterson, and S. Minshall, J. Appl. Phys. 27, 291 (1956) 